\title{
Produção de frutos para uso medicinal em Bromelia antiancatha (caraguatá) : fundamentos para um extrastivismo sustentável
}

\author{
FILIPPON, S. ${ }^{1 *}$; FERNANDES, C.D. ${ }^{1}$; FERREIRA, D.K. ${ }^{1}$; DUARTE, A.S. ${ }^{1}$; REIS, M.S. ${ }^{2}$ \\ ${ }^{1}$ Centro de Ciências Agrárias, ${ }^{2}$ Centro de Ciências Agrárias, Departamento de Fitotecnia, Núcleo de Pesquisas \\ em Florestas Tropicais - NPFT/UFSC, Rodovia Admar Gonzaga, 1346, Itacorubi, CEP: 88034-000, Florianópolis- \\ Brasil *samabio82@gmail.com
}

\begin{abstract}
RESUMO: Entre as várias espécies que têm sido utilizadas como fontes de subprodutos florestais estão a Bromelia antiacantha, espécie nativa da Mata Atlântica com grande potencial de uso com características alimentícias, ornamentais, industriais e farmacológicas. Os frutos da espécie são utilizados tradicionalmente no Planalto Norte Catarinense na confecção de xaropes para tratamento de males das vias respiratórias. Neste contexto, objetivou-se quantificar a produção de frutos e fundamentar estratégias para possível manejo de populações naturais de $B$. antiacantha. Foram acompanhadas 39 infrutescências de Janeiro/2008 a Agosto/2008 distribuídas em área de mata secundária na FLONA de Três Barras, SC. Nesta mesma área, indivíduos da espécie foram acompanhados através de estudos demográficos de 2001 a 2008. As infrutescências apresentaram em média $0,68 \mathrm{~m}$ de comprimento, número médio de 187 frutos/infrutescência, o diâmetro médio dos frutos foi de $1,9 \mathrm{~cm}$ e o peso médio das infrutescências de $3,6 \mathrm{~kg}$. A média de frutos aproveitáveis foi de 157 frutos/infrutescência totalizando $2,5 \mathrm{~kg}$. A safra estimada para 2005 foi de $146 \mathrm{~kg}$ de frutos ha-1 e $80 \mathrm{~kg}$ de frutos ha-1 para 2008, e a renda líquida a partir da produção de xarope foi estimada em $\mathrm{R} \$ 1168,00$ por hectare, por ano. Os resultados mostraram que o manejo de $B$. antiacantha consiste em atividade economicamente interessante e que esta possibilidade, além de complementar a renda de comunidades locais onde a espécie se faz presente, também amplia o valor das áreas com cobertura florestal.
\end{abstract}

Palavras-chave: manejo sustentável, PFNM, plantas medicinais

ABSTRACT: Fruit production for medicinal use in Bromelia antiacantha ("caraguatá"): foundations for sustainable extraction. Among the large number of species that have been used as sources of forest byproducts is Bromelia antiacantha, a species native to the Atlantic Forest and that has great potential of use with nourishing, ornamental, industrial and pharmacological characteristics. The fruits of this species are traditionally used in the Northern Plateau of Santa Catarina State, Brazil, in the preparation of syrup for the treatment of respiratory disorders. In this context, the aim of this study was to quantify the production of fruits and to find strategies for the possible management of natural populations of $B$. antiacantha. A total of 39 inflorescences were observed from January/2008 to August/2008 distributed in a secondary forest area at FLONA (National Forest), Três Barras, Santa Catarina State. In this same area, $B$. antiacantha individuals were accompanied by demographic studies from 2001 to 2008 . The inflorescences presented on average $0.68 \mathrm{~m}$ of length, mean number of 187 fruits/inflorescence, mean diameter of fruits of $1.9 \mathrm{~cm}$ and mean weight of inflorescences of $3.6 \mathrm{~kg}$. The mean number of usable fruits was 157 fruits/inflorescence and the mean weight of these fruits was $2.5 \mathrm{Kg}$. The estimated harvest for 2005 was $146 \mathrm{Kg}_{\text {of fruits ha-1 }}$ and for $2008,80 \mathrm{~kg}$ of fruits ha-1, and the net income from the syrup production could reach $R \$ 1168.00$ per hectare per year. The results showed that the management of $B$. antiacantha consists in an economically interesting activity and that this possibility complements the income of local communities where the species occurs, besides increasing the value of forest areas.

Key words: sustainable management, NTFP, medicinal plants

Recebido para publicação em 18/10/2010

Aceito para publicação em 27/01/2012

Rev. Bras. PI. Med., Botucatu, v.14, n.3, p.506-513, 2012. 


\section{INTRODUÇÃO}

O extrativismo de Produtos Florestais Não Madeireiros (PFNM) tem tido papel importante na história econômica das florestas tropicais (Schroth, 2004). Estes produtos têm sido explorados pelas populações humanas para uso de subsistência através de centenas de anos. Durante as duas últimas décadas os PFNM obtidos de plantas, incluindo sementes, flores, frutos, folhas, raízes, látex, resinas e outras partes não madeiráveis têm ganhado muita atenção nos ciclos da conservação já que o mercado crescente dos produtos naturais, em particular os de plantas medicinais, tem resultado na extração de volumes incríveis das populações naturais (Reis, 1996; Reis et al., 2003; Ticktin, 2004).

Entretanto, apesar da importância sócioeconômica, a exploração indiscriminada de recursos vegetais como o extrativismo predatório dos frutos, tem contribuído para a extinção de populações locais de varias espécies utilizadas tradicionalmente na medicina popular (Castro, 2003). Além da redução de áreas, o extrativismo e até mesmo as mudanças no uso da terra, podem comprometer a diversidade genética principalmente pelo efeito sobre as populações selvagens (Alexiades \& Shanley, 2004). Além disso, a erosão genética também se mostrou um risco para espécies como a pupunha (Bactris gasipaes), ginseng brasileiro (Pfaffia glomerata), carqueja (Baccharis trimera), espécies estas também submetidas ao forte extrativismo (Alexiades \& Shanley, 2004).

O manejo de frutos implica em práticas que utilizam base científica e/ou tradicional. Esta iniciativa pode gerar empregos, distribuição de renda, baixo impacto sócio-ambiental pelo uso de recurso florestal comercialmente valioso e ainda, aumentar do valor da floresta em pé (Rocha, 2004).

Contudo, para que esta prática extrativista seja sustentável ao longo do tempo, torna-se necessário obter e monitorar algumas informações ecológicas para avaliar o potencial de manejo do produto (Fantini et al., 1992; Reis, 1996). Para ser sustentável, o manejo/extrativismo precisa estar baseado no conhecimento da biologia reprodutiva, na distribuição e abundância das espécies (Fantini et al., 1992; Reis, 1996; Reis et al., 2000; Wong, 2000; Reis et al., 2003). Além disso, segundo Wong (2000), o acesso aos PFNM nos trópicos é relativamente novo e possui poucos estudos, portanto, como consequência, as metodologias de manejo têm sido desenvolvidas por pesquisadores de forma isolada em resposta a circunstâncias e peculiaridades dos locais em estudo.

Os produtos florestais não são somente recursos naturais utilizados para atender às necessidades de subsistência, ou simples recursos econômicos negociados entre os diferentes tipos de atores sociais. Segundo Alexiades \& Shanley (2004), estes recursos são também incorporados na vida política, institucional e cultural das pessoas envolvidas na coleta e consumo. Muitos destes produtos têm sido explorados pelas populações humanas para o uso de subsistência através de centenas de anos, principalmente com finalidades medicinais (Ticktin, 2004).

Inúmeras espécies medicinais e frutíferas de importância extrativista são encontradas na Mata Atlântica. Entre essas espécies está Bromelia antiacantha, Bromeliaceae terrestre, nativa da Mata Atlântica, com grande potencial de uso com características medicinais, alimentícias, ornamentais e industriais - fabricação de fibras para tecidos; além de cordoaria e fabricação de sabão a partir dos frutos (Reitz, 1983; Barros \& Souza, 1995; Haverroth, 1997; Mercier \& Yoshida, 1998), reunindo assim, em única espécie, um potencial múltiplo.

Segundo Reitz (1983), a espécie ocorre nos estados do Espírito Santo, Rio de Janeiro, São Paulo, Paraná, Santa Catarina e Rio Grande do Sul. B. antiacantha possui hábito terrestre, com até $3 \mathrm{~m}$ de altura. Desenvolve-se principalmente em solos muito úmidos das florestas de restinga e de vegetação secundária formando sempre densos agrupamentos de forma descontínua pelos diversos ambientes em que ocorre (Reitz, 1983) apresentando características próprias de estrutura espacial (densidade, dispersão, distribuição) e de dinâmica populacional (Reitz, 1983; Santos, 2001; Filippon, 2009).

A utilização na medicina popular é descrita desde a década de 1940, apresentando propriedades anti-helmínticas, antitussígeno e no tratamento de cálculos renais (Reitz, 1983). A ação expectorante e nas infecções respiratórias, além de recomendações para o tratamento de asma e bronquite, foi relatada por Ferro \& Jorge (1993); Reis et al. (2010) e Filippon et al. (2011). Os mesmos frutos são tidos como antihelmínticos (Reitz, 1983; Filippon, 2009) e o sumo é utilizado ainda para limpar feridas (Reitz, 1983).

Estudo etnobotânico realizado na comunidade Passa Quatro, localizada em Turvo, interior do Paraná, mostrou que agricultores utilizam os frutos de $B$. antiacantha para a confecção de xaropes para tratamento de doenças do sistema respiratório (Pacheco et al., 2001). Indicação semelhante foi obtida por Caffer (2005) na região de Caçador - SC, e por Filippon (2009) na comunidade da Campininha, município de Três Barras, SC.

Esta espécie tem despertado interesse de pesquisadores (Santos, 2001; Santos et al., 2004; Brehmer, 2005; Santos, 2006; Duarte et al., 2007; Zanella, 2009; Filippon, 2009, Reis et al., 2010, Zanella et al., 2011) pelo uso dos frutos no combate a doenças do trato respiratório e pelo potencial de manejo e domesticação para obtenção de frutos, 
principalmente no Planalto Norte Catarinense (Reis et al., 2010; Filippon et al., 2011).

Apesar do uso tradicional de $B$. antiacantha, ainda existem poucos estudos no sentido da caracterização demográfica e produção de frutos da espécie (Duarte et al., 2007; Filippon, 2009). Desta forma, este estudo objetivou quantificar a produção de frutos visando fundamentar estratégias para extração sustentável de frutos em populações naturais de $B$. antiacantha.

\section{MATERIAL E MÉTODO}

Neste estudo foram acompanhadas 39 infrutescências (cachos) de Janeiro/2008 a Agosto/ 2008 distribuídas aleatoriamente em uma área de mata secundária na Floresta Nacional de Três Barras (FLONA) Município de Três Barras, SC.

Nesta área, foram realizados estudos demográficos entre 2001 e 2008 (Duarte et al., 2007; Filippon, 2009) em nove parcelas permanentes (800 $\mathrm{m}^{2}$ cada). Nestas parcelas, todas as plantas da espécie foram contadas e caracterizadas quanto ao estádio fenológico, sendo que foram consideradas reprodutivas as plantas que apresentavam inflorescência ou infrutescência. As avaliações foram realizadas em 2001 (Dezembro); 2002 (Novembro); 2003 (Dezembro); 2005 (Fevereiro) e 2008 (Janeiro).

A caracterização das infrutescências foi realizada através da medição do comprimento da infrutescência, da altura de inserção do escapo na roseta e contagem do número de frutos. Além disso, foram tomados aleatoriamente seis frutos de cada infrutescência e determinados o comprimento e o diâmetro, o que foi feito com auxílio de paquímetro. Esse processo foi realizado até o amadurecimento de todas as infrutescências, sendo que na primeira semana de Junho, as primeiras infrutescências maduras foram coletadas.

Depois de coletados, os cachos foram ensacados, identificados e levados ao Núcleo de Pesquisas em Florestas Tropicais (NPFT) no Departamento de Fitotecnia do Centro de Ciências Agrárias da Universidade Federal de Santa Catarina. Os cachos foram então pesados, os frutos selecionados e separados em frutos aproveitáveis e frutos descarte. Foram considerados descartes aqueles frutos não sadios (com manchas, não desenvolvidos completamente ou danificados) e aproveitáveis aqueles que poderiam ser utilizados para possível produção de xarope ou comercialização.

Todos os frutos (aproveitáveis e descarte) foram pesados e os aproveitáveis foram armazenados em câmara fria $\left( \pm 10^{\circ} \mathrm{C}\right)$. Com estes dados estimouse a produtividade de frutos por planta.

Os indivíduos dos quais as infrutescências foram obtidas também foram avaliados, caracterizandose o número total de folhas aparentes e o comprimento médio de três folhas.

Os dados obtidos foram processados visando a obtenção de estatísticas descritivas (Sokal \& Rohlf, 1995).

\section{RESULTADO E DISCUSSÃO}

As infrutescências apresentaram em média $0,68 \pm 0,09 \mathrm{~m}$ de comprimento (Tabela 1 ), sendo que para esta variável, houve variação de 0,51 a 0,84 m. A altura mínima de inserção do escapo na roseta foi de $0,08 \mathrm{~m}$, a máxima foi $0,16 \mathrm{~m}$, e a média obtida foi de $0,11 \mathrm{~m}$. O número médio de frutos por infrutescência foi de 187, observando-se expressiva variação de 50 a 320 frutos/infrutescência (Tabela 1). Duarte et al. (2007), empregando uma amostra de 10 infrutescências, obtiveram resultados semelhantes em trabalho realizado no mesmo local, onde a média de comprimento da infrutescência foi de 0,66 m e o número médio de frutos foi 166,2 frutos/ infrutescência.

O diâmetro médio dos frutos variou de 0,8 $\mathrm{cm}$ a 2,65 cm, com média de $1,9 \mathrm{~cm}$ (desvio padrão $s=0,37$ ) (Tabela 1). Como esperado, ao longo do período de avaliação houve aumento de diâmetro. A variação no incremento em diâmetro $(\mathrm{cm}$ por quinzena) dos frutos foi avaliada através de regressão sendo que o modelo de melhor ajuste para explicar o comportamento dos frutos foi o quadrático com constante $\left(y=0,61+0,34 x-0,02 x^{2} ; R^{2}=0,90\right)$. $O$ incremento total no diâmetro dos frutos variou de 0,33 a $1,40 \mathrm{~cm}$, sendo que a média do incremento total do diâmetro dos frutos foi de $0,79 \pm 0,10 \mathrm{~cm}$ por fruto. $A$ média de incremento por fruto foi de $0,12 \pm 0,02 \mathrm{~cm}$.

TABELA 1. Características de infrutescências (cachos) e frutos em uma população natural de Bromelia antiacantha sob cobertura de Floresta Ombrófila Mista, Três Barras, SC. UFSC/RGV/NPFT/ 2008.

\begin{tabular}{lccccc}
\hline & $\begin{array}{c}\text { Comprimento/ } \\
\text { infrutescência }(\mathbf{m})\end{array}$ & $\begin{array}{c}\text { Altura de inserção } \\
\text { do escapo }(\mathbf{m})\end{array}$ & $\begin{array}{c}\text { Número } \\
\text { de frutos }\end{array}$ & $\begin{array}{c}\text { Comprimento } \\
\text { do fruto }(\mathbf{c m})\end{array}$ & $\begin{array}{c}\text { Diâmetro do } \\
\text { fruto }(\mathbf{c m})\end{array}$ \\
\hline Média & 0,68 & 0,11 & 187 & 3,7 & 1,9 \\
Desvio padrão (s) & 0,09 & 0,002 & 71,7 & 0,51 & 0,37 \\
Amplitude & $0,5-0,84$ & $0,08-0,16$ & $50-320$ & $2,9-4,9$ & $0,8-2,65$ \\
\hline
\end{tabular}

Rev. Bras. PI. Med., Botucatu, v.14, n.3, p.506-513, 2012. 
Uma correlação negativa moderada e significativa $(R=-0,37 ; p=0,0000)$ foi evidenciada para este parâmetro em relação ao período de avaliação, corroborando desta forma, com os resultados obtidos nas análises das médias de diâmetro dos frutos.

Ao longo das avaliações em campo observou-se que o número de frutos sadios diminuiu de maneira constante. Das 39 infrutescências observadas, somente em cinco não foi registrada a perda de frutos, sendo que foi calculada média de $3,17 \pm 1,20$ frutos/infrutescência perdidos a cada 15 dias (Tabela 2). Apesar de ser uma perda aparentemente baixa, quando somados todos os frutos perdidos a média foi de 31,5 $\pm 11,8$ frutos/ infrutescência. As perdas iniciaram já na primeira quinzena após a avaliação inicial (4,70 $\pm 5,7$ frutos).

Nas quinzenas $4,5,6$ e 7 , mais precisamente de Abril a início de Junho/2008, ocorreram perdas consecutivas bastante expressivas. Neste período foi observada com bastante frequência a presença de insetos como formigas e pequenos besouros (Curculionidae). Os frutos começaram a escurecer e a liberar uma substância gelatinosa, além disso, foram encontrados muitos frutos abertos e completamente despolpados (ocos). Também foram, ao final dos trabalhos de campo (Julho-Agosto/2008), observados frutos consumidos pela fauna e caídos próximos às plantas.

A presença de vários Curculionidae (Coleoptera) foi registrada constantemente nas flores em antese de Aechmea beeriana por Nara \& Weber
(2002). Os pesquisadores registraram ainda a presença de larvas de Diptera que predavam estiletes, filetes e anteras, inviabilizando a polinização, e larvas de Coleoptera que se alimentavam das sementes causando a destruição parcial ou completa. Segundo esses autores, cerca de $30 \%$ do total de 62 infrutescências de $A$. beeriana foram destruídas total ou parcialmente, seja pela intensa predação observada nas flores pelas larvas de drosofilideos, seja pela ação das larvas dos coleópteros nos frutos em desenvolvimento.

O peso médio das infrutescências foi de 3,6 $\mathrm{kg}$ com variação de 0,9 a 6,3 kg/infrutescência. Após a seleção dos frutos aproveitáveis (Tabela 3 ) obtevese média de 157 frutos/infrutescência (variação de 32 a 315 frutos/infrutescência). Estimando a produtividade de frutos da mesma espécie, Duarte et al. (2007) observaram peso médio da infrutescência de $2,5 \mathrm{~kg}$, média idêntica a obtida para o peso total dos frutos aproveitáveis no presente trabalho. O peso médio dos frutos descarte foi $0,6 \mathrm{~kg}$ sendo média de 77 frutos descarte/infrutescência (variação de 15 a 206).

Os indivíduos dos quais as infrutescências foram coletadas apresentaram em média 80 folhas/ indivíduo e comprimento médio de folha de $2,5 \mathrm{~m}$, valor este, próximo ao registrado por Duarte et al. (2007), onde a média de comprimento de folha foi de $2,57 \mathrm{~m}$.

Não foi observado incremento e, consequentemente, troca de classe para os indivíduos reprodutivos, sendo que este fato corrobora com o

TABELA2. Número de frutos, comprimento e o diâmetro médios dos frutos por infrutescência de Bromelia antiacantha por quinzena. UFSC/RGV/NPFT/ 2008.

\begin{tabular}{ccccc}
\hline Período & NMFT & CMFT $(\mathbf{c m})$ & DMFT $(\mathbf{c m})$ & $\mathbf{n}$ \\
\hline Início & $187,1 \pm 23,2$ & $3,7 \pm 0,2$ & - & 39 \\
1a quinzena & $182,4 \pm 25,0$ & $3,7 \pm 0,2$ & - & 39 \\
2a quinzena & $182,6 \pm 24,3$ & $3,7 \pm 0,2$ & - & 39 \\
3a quinzena & $180,0 \pm 25$ & $3,7 \pm 0,2$ & $1,5 \pm 0,09$ & 39 \\
4a quinzena & $176,0 \pm 25,1$ & $3,7 \pm 0,2$ & $1,6 \pm 0,09$ & 39 \\
5a quinzena & $172,0 \pm 25,4$ & $3,7 \pm 0,1$ & $1,8 \pm 0,1$ & 39 \\
6a quinzena & $168,1 \pm 25,4$ & $3,7 \pm 0,1$ & $2,0 \pm 0,1$ & 39 \\
7a quinzena & $162,2 \pm 25,7$ & $3,8 \pm 0,1$ & $2,0 \pm 0,08$ & 39 \\
8a quinzena & $160,6 \pm 25,7$ & $3,8 \pm 0,1$ & $2,1 \pm 0,1$ & 39 \\
9a quinzena & $173,7 \pm 29,8$ & $3,7 \pm 0,2$ & $2,0 \pm 0,1$ & 31 \\
10a quinzena & $182,6 \pm 34,2$ & $3,6 \pm 0,2$ & $2,0 \pm 0,09$ & 27 \\
11a quinzena & $204,5 \pm 51,9$ & $3,7 \pm 0,3$ & $2,0 \pm 0,13$ & 21 \\
12a quinzena & $211,5 \pm 47,3$ & $3,7 \pm 0,4$ & $2,0 \pm 0,1$ & 11 \\
\hline Média & $180,29 \pm 9,0$ & $3,7 \pm 0,02$ & $1,9 \pm 0,2$ & - \\
\hline
\end{tabular}

NMFT= número médio de frutos por infrutescência; CMFT= comprimento médio dos frutos \pm intervalo de confiança da média (IC); DMFT= diâmetro médio dos frutos \pm intervalo de confiança da média (IC); $n$ = número de infrutescências avaliadas.

Rev. Bras. PI. Med., Botucatu, v.14, n.3, p.506-513, 2012. 
TABELA 3. Produção de frutos e características biométricas de plantas reprodutivas $(n=39)$ de Bromelia antiacantha; UFSC/RGV/NPFT/ 2008.

\begin{tabular}{lccccccc}
\hline & $\begin{array}{c}\text { PI } \\
(\mathbf{k g})\end{array}$ & $\begin{array}{c}\text { Número } \\
\text { FA }\end{array}$ & $\begin{array}{c}\text { Peso FA } \\
\mathbf{( k g})\end{array}$ & $\begin{array}{c}\text { Número } \\
\text { FD }\end{array}$ & $\begin{array}{c}\text { Peso FD } \\
(\mathbf{k g})\end{array}$ & $\begin{array}{c}\text { Número } \\
\text { folhas }\end{array}$ & $\begin{array}{c}\text { Comprimento } \\
\text { folha }(\mathbf{m})\end{array}$ \\
\hline Média & 3,6 & 157 & 2,5 & 77 & 0,6 & 80 & 2,53 \\
Desvio padrão (s) & 1,3 & 78 & 1,17 & 40 & 0,29 & 9 & 0,23 \\
Amplitude & $0,99-6,3$ & $32-315$ & $0,44-4,30$ & $15-206$ & $0,09-1,44$ & $62-99$ & $2-3$ \\
\hline
\end{tabular}

$\mathrm{PI}=$ Peso da infrutescência; FA = Frutos aproveitáveis; FD = Frutos descarte.

descrito por Andrade et al. (2009), em trabalho com Tillandsia stricta. Ou seja, o pesquisador menciona que à medida que indivíduo incrementa a biomassa, a alocação relativa na reprodução diminui, entendendo-se desta maneira que o indivíduo que está em fase reprodutiva terá menor incremento. Desta forma, ainda segundo o autor, um indivíduo pequeno aloca porcentagem muito maior de recurso para reprodução e mesmo assim o investimento é modesto quando comparado ao de um indivíduo maior, que aloca porcentagem menor de recurso e obtém melhores resultados.

Tendo-se como base estudos demográficos realizados de 2001 a 2008 na mesma área, considerando-se diferentes cenários para exploração dos frutos, 25, 50 e $75 \%$ das infrutescências e admitindo-se que a média de peso total dos frutos aproveitáveis observada neste estudo foi de $2,5 \mathrm{~kg}$, pode-se estimar a produtividade para cada ano bem como a produção potencial de xarope (Tabela 4). A estimativa de renda foi calculada com base na receita fornecida por Pacheco et al. (2001), onde $0,5 \mathrm{~kg}$ de fruto rende $2 \mathrm{~L}$ de xarope com custo de $R \$ 5,00$ podendo ser vendido o litro por $R \$ 7,00$. Desta forma, em 2001, por exemplo, a renda líquida chegaria a $R \$$ 1168,00 por hectare por ano (R\$ 97,33 / mês). Os cenários propostos consideram a possibilidade da proporção remanescente ser consumida pela fauna e ainda garantir a regeneração natural da espécie.

Em estimativa semelhante, Duarte et al. (2007) estimaram para o ano de 2002 produtividade de $152,5 \mathrm{~kg}$ de frutos de $B$. antiacantha por hectare. Segundo a autora, que utilizou receita diferente como base dos cálculos, a estimativa foi de produção de 610 litros de xarope o qual poderia ser vendido por até $R \$ 7,00$ o litro, gerando renda de $R \$ 1220,00$ por hectare por ano, logo, $R \$ 101,60$ por mês. Segundo informações do Laboratório Catarinense (2011), um

TABELA 4. Estimativa de safra de frutos de Bromelia antiacantha para exploração de $25 \%, 50 \%$ e $75 \%$ dos indivíduos reprodutivos. UFSC/RGV/NPFT/ 2008.

\begin{tabular}{|c|c|c|c|c|c|}
\hline $\begin{array}{c}\text { Ano de } \\
\text { avaliação }\end{array}$ & $\begin{array}{c}\text { Número médio de } \\
\text { indivíduos reprodutivos } \\
\text { ha-1 }^{-1} \text { (NMIR) }\end{array}$ & $\begin{array}{l}\text { Estimativa } \\
\text { de safra } \\
\left(\text { kg ha-1) }^{-1}\right.\end{array}$ & $\begin{array}{l}\text { Estimativa de } \\
\text { produção de } \\
\left.\text { xarope (L ha }{ }^{1}\right)\end{array}$ & $\begin{array}{l}\text { Estimativa de renda } \\
\text { bruta a partir de } \\
\left.\text { xarope ( } R \$ \text { ha }^{1}\right)\end{array}$ & $\begin{array}{l}\text { Estimativa de renda } \\
\text { líquida a partir de } \\
\text { xarope ( } R \$ \text { ha }^{1} \text { ) }\end{array}$ \\
\hline \multicolumn{6}{|c|}{$25 \%$} \\
\hline 2001 & 117 & 73 & 293 & 2047,50 & 585,00 \\
\hline 2003 & 67 & 42 & 168 & 1172,50 & 335,00 \\
\hline 2005 & 117 & 73 & 293 & 2047,50 & 585,00 \\
\hline 2008 & 64 & 40 & 160 & 1120,00 & 320,00 \\
\hline \multicolumn{6}{|c|}{$50 \%$} \\
\hline 2001 & 117 & 146 & 584 & 4088,00 & 1168,00 \\
\hline 2003 & 67 & 84 & 336 & 2352,00 & 672,00 \\
\hline 2005 & 117 & 146 & 584 & 4088,00 & 1168,00 \\
\hline 2008 & 64 & 80 & 320 & 2240,00 & 640,00 \\
\hline \multicolumn{6}{|c|}{$75 \%$} \\
\hline 2001 & 117 & 219 & 878 & 6142,50 & 1755,00 \\
\hline 2003 & 67 & 126 & 503 & 3517,50 & 1005,00 \\
\hline 2005 & 117 & 219 & 878 & 6142,50 & 1755,00 \\
\hline 2008 & 64 & 120 & 480 & 3360,00 & 960,00 \\
\hline
\end{tabular}

Rev. Bras. Pl. Med., Botucatu, v.14, n.3, p.506-513, 2012. 
xarope expectorante já conhecido no mercado era, em 2001, vendido ao consumidor final no preço máximo de $\mathrm{R} \$ 7,04$ o frasco com $150 \mathrm{ml}$. Tendo-se por base estes valores e a produtividade de $B$. antiacantha em 2001, poderiam ser produzidos cerca de 3.854 frascos de xarope que, vendido pelo valor acima citado, gerariam uma renda bruta de $\mathrm{R} \$$ $27.134,97$. Entretanto, neste caso não estão inclusos os custos com a legalização do produto, já que se trataria de novo xarope no mercado.

Considerando a produção e a possível renda obtida somente com os frutos da espécie, percebese que o manejo de $B$. antiacantha consiste em atividade economicamente interessante. Comparandose essa produção com a de culturas tradicionais verifica-se que o trabalho com a espécie pode ser economicamente vantajoso. De acordo com a produção agrícola de 2001 no estado de Santa Catarina, foram produzidos em média $2.687 \mathrm{~kg} \mathrm{ha}^{-1}$ de soja no estado (EPAGRI/CEPA, 2011), os quais foram vendidos com preço médio de $\mathrm{R} \$ 0,37$ por $\mathrm{kg}$, resultando na renda bruta de $\mathrm{R} \$ 997,68 \mathrm{ha}^{-1}$ ano-1 (R\$ 83,14 ha $^{-1}$ mês $^{-1}$ ) (valores estes calculados a partir do preço médio da saca de $60 \mathrm{~kg}$ em 2001 fornecidos pela EPAGRI/CEPA 2011). A produção de milho no mesmo ano foi de $4.404 \mathrm{~kg} \mathrm{ha}^{-1} \mathrm{ano}^{-1}$ (EPAGRI/CEPA, 2011 ), sendo que o preço médio de venda foi $R \$ 0,16$ kg. Logo, a renda bruta obtida em 2001 com esta cultura foi de $\mathrm{R} \$ 686,14 \mathrm{ha}^{-1} \mathrm{ano}^{-1}$. O custo total da lavoura de soja em 2001 em Santa Catarina foi de R\$ 722,80 e para o milho, produzido com baixa tecnologia, foi de R\$499,28 (EPAGRI/CEPA, 2011). Subtraindo-se os gastos com preparação do solo, sementes, insumos, maquinário e mão de obra utilizados nestas duas culturas, o lucro líquido com a produção de soja, foi de $\mathrm{R} \$ 274,88 \mathrm{ha}^{-1} \mathrm{ano}^{-1}$ ( $\mathrm{R} \$$ 22,91 ha $^{-1}$ mês $^{-1}$ ) e o lucro com o milho foi de $\mathrm{R} \$$ 186,86 ha-1 ano-1 (R\$ 15,57 ha-1 mês-1).

Comparando-se os valores obtidos com culturas anuais aos estimados para um hectare de B. antiacantha com possibilidade de se coletar $50 \%$ dos cachos para produção de xarope verifica-se que o lucro obtido é superior ao das culturas anuais (Tabela 4). É importante considerar que, embora o valor da produção de xarope seja superior ao de culturas anuais, o potencial do mercado para absorver a totalidade da produção deste xarope ainda não é conhecido. Além disso, há a necessidade de mais estudos em relação à comercialização bem como dos custos de produção e legalização do produto.

Comparando-se ainda a estimativa de lucros obtidos a partir do manejo de $B$. antiacantha com os obtidos com a Pupunha (Bactris gasipaes) também se verifica que $B$. anticantha possui uma possível maior margem de lucro. O custo de implantação de uma lavoura de pupunha é de $\mathrm{R} \$ 6.000,00 \mathrm{ha}^{-1}$, o primeiro corte não chega a pagar todos os gastos de implantação ( $R \$ 4.000,00$ ha $^{-1}$ ) e que nos cortes subsequentes a renda será de $\mathrm{R} \$ 7200,00 \mathrm{ha}^{-1}$ em 12 anos, ou seja, R\$ 600,00 ha-1 ano (INPA, 2009).

As atividades humanas, especialmente as práticas de extrativismo e manejo influenciam na prospecção do uso dos produtos florestais não madeireiros pelo impacto causados na floresta em vários níveis (Sinha \& Bawa, 2002). De acordo com Homma (1996), a sustentabilidade do extrativismo está relacionada não somente à oferta de estoques extrativos, mas também com políticas de desenvolvimento tecnológico, ecológico, científico, demanda de produtos e correntes migratórias. Segundo o autor, o extrativismo tende a seguir quatro fases características de um ciclo econômico, a expansão, a extração, o declínio e a queda da extração (substituição/domesticação). Entretanto, este conceito está limitado somente à extração dos produtos considerando o enriquecimento, o cultivo, a criação ou o beneficio, como um sistema domesticado.

Neste contexto, são necessários estudos adicionais para avaliação do impacto da extração sobre a diversidade genética e regeneração natural da espécie, bem como sobre a disponibilidade deste recurso para a fauna, visando o estabelecimento efetivo de estratégias sustentáveis de manejo e mercado já que o que se busca além do benefício às comunidades locais através de renda é a valorização dos recursos que a floresta pode oferecer.

\section{AGRADECIMENTO}

Os autores agradecem à equipe do ICMBio da Floresta Nacional de Três Barras pelo suporte durante os estudos no local, à equipe do Núcleo de Pesquisas em Florestas Tropicais da Universidade Federal de Santa Catarina pelo auxílio nas atividades de campo e análise dos dados, à Capes e ao CNPq pela concessão das bolsas durante o desenvolvimento do trabalho.

\section{REFERÊNCIA}

ALEXIADES, M.N.; SHANLEY, P. Forest products, livelihoods and conservation: case studies of non-timber forest product systems. In: ALEXIADES, M.N.; SHANLEY, P. (Eds.). Productos forestales medios de subsistencia y conservación: estudios de caso sobre sistemas de manejo de productos forestales no maderables. Indonésia: Centro para La Investigación Forestal Internacional. v.3, 2004. 499p.

ANDRADE, S.C.S. et al. Influência do tamanho do rameto sobre a alocação de biomassa na reprodução sexuada de Tillandsia stricta (Bromeliaceae.) Relatórios. Disponível em: <http://www.ib.unicamp.br/profs/fsantos/ relatorios/ne313-c2.pdf>. Acesso em: 06 fev. 2009.

Rev. Bras. PI. Med., Botucatu, v.14, n.3, p.506-513, 2012. 
BARROS, I.P.I.; SOUZA, P.L. Coleta de germoplasma de Bromelia antiacantha (Bromeliaceae) no Rio Grande do Sul. In: SIMPÓSIO NACIONAL DE RECURSOS GENÉTICOS VEGETAIS, 1., 1995, Campinas. Anais... Campinas: EMBRAPA-CENARGEN, 1995. p.17.

BREHMER, J.S. Estudo de extratos de plantas medicinais no desenvolvimento do tumor ascítico de Ehrlich. 2005. 60p. Dissertação (Mestrado - Ciências Farmacêuticas) - Universidade do Vale do Itajaí, Itajaí. CAFFER, M.M. Caracterização do conhecimento de populações locais sob a diversidade de RGV em remanescentes de FOM. 2005. 104p. Dissertação (Mestrado em Recursos Genéticos Vegetais) Universidade Federal de Santa Catarina, Florianópolis. CASTRO, A.H.F. Aspectos da propagação, ecofisiologia e fitoquímica de Byrsonima verbascifolia Rich. ex A. Juss.: uma espécie medicinal do cerrado. 2003. 130p. Tese (Doutorado em Fisiologia Vegetal) - Universidade Federal de Lavras, Lavras.

DUARTE, A.S. et al. Estrutura demográfica e produção de frutos de Bromelia antiacantha Bertol. Revista Brasileira de Plantas Medicinais, v.9, n.3, p.106-12, 2007.

EPAGRI/CEPA. Empresa de Pesquisa Agropecuária e Extensão Rural de Santa Catarina / Centro de Socioeconomia e Planejamento Agrícola. Disponivel em: <http://www.cepa.epagri. sc.gov.br>. Acesso em: $21 \mathrm{dez}$. 2011.

FANTINI, A.C.; REIS, M.S.; GUERRA, M.P. Sustained yield management in tropical Forest: a proposal based on the autoecology of the species. Sellowia, v.42, p.25-33, 1992. FERRO, V.O.; JORGE L.I.F. Reconhecimento da espécie Bromelia antiacantha (Bertol.): características botânicas e fitoquímicas. Revista de Farmácia e Bioquímica da Universidade de São Paulo, v.29, n.2, p.69-72, 1993. FILIPPON, S. Aspectos da demografia, fenologia e uso tradicional do Caraguatá (Bromelia antiacantha Bertol.) no Planalto Norte Catarinense. 2009. 104p. Dissertação (Mestrado em Recursos Genéticos Vegetais) Universidade Federal de Santa Catarina, Florianópolis. FILIPPON, S. et al. Bromelia antiacantha. In: CORADIN, L.; REIS, A.; SIMINSKI, A. (Orgs.). Espécies nativas da flora brasileira de valor econômico atural ou potencial: plantas do futuro - Região sul. Brasília: Ministério do Meio Ambiente. v.1, 2011. p.568-77.

HAVERROTH, M. Kaingang - um estudo etnobotânico. O uso e a classificação das plantas na área Indígena Xapecó. 1997. 192p. Dissertação (Mestrado em Antropologia) - Curso de Pós-graduação em Antropologia Social, Universidade Federal de Santa Catarina, Florianópolis.

HOMMA, A.K.O. Extrativismo vegetal na Amazonida: limites e possibilidades. In: EXTRATIVISMO NAAMOZINA BRAILEIRA PERSPECTIVAS SOBRE O DESENVOLVIMETNO REGIONAL, 18., 1996. Montevideo. Compendio... Montevideo: Unesco, 1996, p.35-61.

INPA. Instituto Nacional de Pesquisasda Amazônia. Disponível em: <www.inpa.gov.br>. Acesso em: 05 dez. 2011.

MERCIER, H.; YOSHIDA, M.K. Bromelian activity leaf tissue of Bromelia antiacantha. Journal of the Bromeliad Society, v.48, n.2, p.6-10, 1998.
NARA, A.K.; WEBBER, A.C. Biologia floral e polinização de Aechmea beeriana (Bromeliaceae) em vegetação de baixio na Amazônia Central. Acta amazônica, v.32, n.4, p.571-88, 2002.

PACHECO, C.V. et al. Plantas da nossa gente. A sabedoria popular no uso de plantas medicinais. Projeto Florestas Medicinais. Guarapuava, 2001, 36p.

REIS, M.S.; MARIOT, A.; STEENBOCK, W. Diversidade e domesticação de plantas medicinais. In: SIMÕES, C.M.O. et al. (Eds.). Farmacognosia - da planta ao medicamento. Florianópolis/Porto Alegre: Editora da UFSC/UFRGS. 2003, p.45-74.

REIS, M.S. Manejo sustentado de plantas medicinais em ecossistemas tropicais. In: DI STASI, L.C. (Org.). Plantas medicinais arte e ciência. São Paulo: Editora da Universidade Estadual Paulista, 1996, p.199-215.

REIS, M.S. et al. Management and conservation of natural populations in Atlantic rain Forest: the case study of palm heart (Euterpe edulis Martius). Biotropica, v.32, n.4B, p.894-902, 2000.

REIS, M.S.R. et al. Uso sustentável e domesticação de espécies da Floresta Ombrófila Mista. In: LIN C.M; AMOROZO, C.M.; KFFURI, C.W. (Orgs.). Agrobiodiversidade no Brasil: experiências e caminhos da pesquisa. Recife: NUPEEA. 2010. p.183-214.

REITZ, R. Bromeliaceas e a malária - bromélia endêmica. Flora ilustrada Catarinense. Itajaí: Herbário Barbosa Rodrigues, 1983. 559p.

ROCHA, E. Potencial ecológico para o manejo de frutos de açaizeiro (Euterpe precatoria Mart.) em áreas extrativistas no Acre, Brasil. Acta Amazônica, v.32, n.2, p.237-350, 2004.

SANTOS, D.S. Biologia reprodutiva de Bromelia antiacantha Bertol. (Bromeliaceae) em uma população natural sob cobertura de Floresta Ombrófila Mista. 2001. 96p. Dissertação (Mestrado em Recursos Genéticos Vegetais) - Universidade Federal de Santa Catarina, Florianópolis.

SANTOS, D.S. et al. Variação no período de germinação de sementes em uma população natural de Bromelia antiacantha Bertol. Revista Brasileira de Plantas Medicinais, v.6, n.3, p.35-41, 2004.

SANTOS, V.N.C. Banana-do-mato: investigação do perfil químico e bioatividade dos frutos maduros de Bromelia antiacantha. 2006. 61p. Trabalho de conclusão de curso (Bacharelado em Biologia) - Universidade do Vale do Itajaí, Itajaí.

SCHROTH, G. et al. Extractive use, management and in situ domestication of a weedy palm, Astrocaryum tucumã, in the central Amazon. Forest Ecology and Management, v.202, p.167-79, 2004.

SINHA, A.; BAWA, K.S. Harvesting techniques, hemiparasites and fruit production in two nontimber forest tree species in south Índia. Forest Ecology and Management, v.168, p.289-300, 2002.

SOKAL, R.R.; ROHLF, F.J. Biometry: the principles and pratice of statistics in biological research. 3.ed. New York: W. H. Freeman and Company, 1995, 887p.

TICKTIN, T. The ecological implications of harvesting nontimber forest products. Journal of Applied Ecology, v.41, p.11-21, 2004.

WONG, J.L.G. The biometrics of non-timber forest product resouce asessment: a review of current 
methodology. Oxford, p.1-109. 2000. Disponível em: $<$ www.etfrn.org/ etfrn/workshop/ntfp/text.pdf>. Acesso em: 19 dez. 2011

ZANELLA, C. Caracterização genética, morfológica e fitoquímica de populações de Bromelia antiacantha

(Bertol.) do Rio Grande do Sul. 2009. 98p. Dissertação
(Mestrado em Genética e Biologia Molecular) Universidade Federal do Rio Grande do Sul, Porto Alegre. ZANELLA, C. et al. Genetic structure and phenotypic variation in wild populations of the medicinal tertraploid species Bromelia antiacantha (Bromeliaceae). American Journal of Botany, v.98, n.9, p.1511-9, 2011. 